\title{
FIBRE BRAGG GRATING FABRICATION IN GERMANOSILICATE FIBRES WITH 244nm FEMTOSECOND LASER LIGHT
}

\author{
A. Canagasabey ${ }^{1}$, J. Canning ${ }^{2}$, J. D. Mills ${ }^{1}$, D. P Banks ${ }^{1}$ and M. Ibsen ${ }^{1}$ \\ ${ }^{1}$ Optoelectronics Research Centre, University of Southampton, \\ Southampton, SO17 1BJ, United Kingdom \\ ${ }^{2}$ Optical Fibre Technology Centre, University of Sydney, \\ Australian Technology Park, Eveleigh, NSW 1430, Australia
}

\begin{abstract}
The ability to fabricate strong and extraordinarily stable Bragg gratings in any type of fibre using femtosecond laser light has attracted much interest in the recent past. The induced index change has been attributed to glass densification brought about by nonlinear multiphoton ionisation resulting in bond breaking, local melting and rapid cooling occurring after optical breakdown by the high-energy femtosecond light.
\end{abstract}

In this paper, a preliminary comparative study between fibre Bragg grating fabrication in germanosilicate fibre using $244 \mathrm{~nm}$ femtosecond source with a repetition rate of $250 \mathrm{kHz}$ and $200 \mathrm{fs}$ pulse duration and a $244 \mathrm{~nm} \mathrm{CW}$ frequency doubled argon-ion source is presented. The reflectivities achieved in both cases were close to identical for similar writing fluences, as were the isochronal annealing profiles. This indicated that despite the very high repetition rate used, the collective heat generated by the femtosecond radiation plays no role in the compaction of the glass matrix. The index change observed in this case is a result of single photon absorption into known defect centres - the laser intensity was insufficient to excite the glass band edge leading to glass compaction. No qualitative difference in index change between $\mathrm{CW}$ and short-pulse excitation of these defects is found.

\section{Introduction}

Since the first time waveguide structures were fabricated in glasses exposed to high energy femtosecond radiation $[1,2]$, interest in using these sources for fibre-based applications requiring permanent index change has grown considerably. Long period fibre gratings have been fabricated using focussed $800 \mathrm{~nm}$ radiation from a Ti:Sapphire source [2], and with a $264 \mathrm{~nm}$ frequency quadrupled Nd:YAG source [3], utilising point-by-point techniques.

More recently, Bragg gratings have been fabricated using the phase mask technique in the Corning smf-28 telecommunications fibre [4] and also in pure silica fibres [5]. Index changes as high as $1.9 \times 10^{-3}$ have been reported in fibres not photosensitive to the $800 \mathrm{~nm}$ light used [5]. Index change in these fibres therefore has been attributed to glass densification brought about by nonlinear multiphoton ionisation resulting in local melting and rapid cooling occurring after optical breakdown by the high energy femtosecond light [1,2]. Extraordinary stability of femtosecond laser fabricated structures in silica based materials has also been reported [6,7].

In this report, a comparative study between fibre Bragg Gratings (FBG) fabricated conventionally using a continuous wave $(\mathrm{CW})$ frequency doubled argon ion source operating at $244 \mathrm{~nm}$ and a titanium sapphire based femtosecond laser source of intensity $5.73 \times 10^{3} \mathrm{Wcm}^{-2}$ is presented.

\section{Experiments and Results}

The output of the femtosecond 800nm Ti:Sapphire laser system was amplified using a regenerative amplifier system before being converted to $488 \mathrm{~nm}$ using an optical parametric amplifier (OPA). The OPA output is then frequency doubled to give the desired $244 \mathrm{~nm}$ photons. This system operates at a repetition rate of $250 \mathrm{kHz}$ and the pulse duration at the output of the frequency doubler is roughly estimated to be 200fs. The average power at $244 \mathrm{~nm}$ was $4.5 \mathrm{~mW}$, which is $\sim 18 \mathrm{~nJ}$ energy per pulse.

FBGs were fabricated using the $244 \mathrm{~nm}$ femtosecond output through a standard phase mask in pristine boron co-doped germanosilicate fibres, PS1250/1500 from FiberCore with core diameter $6.9 \mu \mathrm{m}$, and numerical aperture 0.13. FBGs were also fabricated using a CW frequency doubled argon ion laser source through a phase mask in the same fibre. Two gratings written using the two sources for an identical fluence of $\sim 1380 \mathrm{Jcm}^{-2}$ were isochronally annealed at temperatures of $100^{\circ} \mathrm{C}, 200^{\circ} \mathrm{C}$, $300^{\circ} \mathrm{C}$ and $400^{\circ} \mathrm{C}$.

From Figure 1(a) it can be seen that the annealing behaviour for the femtosecond written grating is comparable to that of the grating written conventionally, using a frequency doubled argon ion laser. This suggests that the femtosecond grating is also a Type 1 grating. Thus the index change in the femtosecond laser written grating is similar to that of the $\mathrm{CW}$ argon ion laser grating, which is purely from single photon absorption into germanium based defect centres in the germanosilicate fibre. Figure 1(b) shows the Bragg grating shift in the two gratings under isochronal annealing with the Bragg grating shifts being near identical. This again strongly suggests that the intensity of the femtosecond light used is below the threshold required for glass compaction through nonlinear 
ionisation and that the mechanisms responsible for index change are no different to that of $\mathrm{CW}$ light exposure.

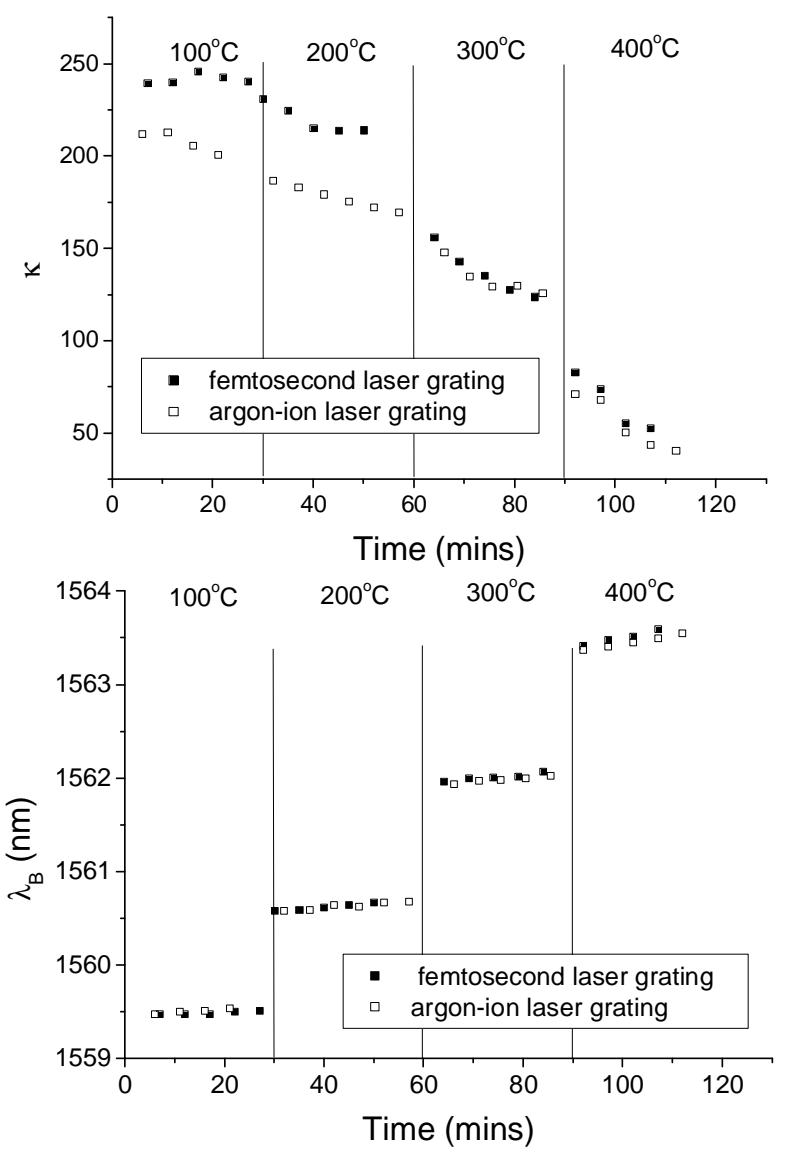

Figure 1(a) Annealing behaviour of the coupling coefficient, kappa $(\kappa)$ of gratings written with femtosecond and CW argon ion lasers. (b) Annealing behaviour of the Bragg wavelength for the same gratings. The slight gradient seen in the Bragg wavelength shift for both gratings, particularly at $300^{\circ} \mathrm{C}$ and $400^{\circ} \mathrm{C}$ temperatures is thought to be due to the temperature instability of the annealing oven.

The mechanisms that lead to structural changes in dielectrics with femtosecond laser irradiation are not completely understood. Nonlinear photon absorption processes, however, are thought to play a major role [8]. These can be categorised into multiphoton ionisation and avalanche ionisation. Multiphoton ionisation occurs as a result of simultaneous absorption of two or more photons by an electron, enabling it to gain sufficient energy to move to the conduction band. Avalanche ionisation on the other hand occurs as a result of progressive absorption of photons by an electron already present in the conduction band. This process, known as Joule's heating enables the electron to gather sufficient energy to ionise another electron in the valence band by collision, bringing it into the conduction band. This 'avalanche effect' can commence as long there is an electron present in the conduction band. This seed electron may come from multiphoton ionisation for high intensity radiation, from thermal excitation or simply from defects in the material. In the case of gratings written with $244 \mathrm{~nm}$ femtosecond light neither of these processes seem to take place despite the presence of germanium defects and femtosecond pulses capable of triggering Joule's heating in the glass matrix. The local intensity is therefore not sufficiently high to allow multiphoton excitation.

In conclusion, an intensity of $5.73 \times 10^{3} \mathrm{Wcm}^{-2}$ of the $244 \mathrm{~nm}$ femtosecond source was insufficient to trigger nonlinear ionisation processes responsible for greater index changes. The threshold intensity required for glass compaction was not reached despite a 2-photon $244 \mathrm{~nm}$ process requiring considerably less intensity than at 800nm: $\sim 2.1 \times 10^{13} \mathrm{Wcm}^{-2}$ for a 5-6-photon process [5]. This indicates insufficient intensity to overcome efficient energy transfer though from single photon excitation of defect sites resulting in conventional Type I gratings. These Type 1 gratings fabricated with femtosecond irradiation displayed similar annealing behaviour to that of gratings fabricated conventionally using CW 244nm argon ion laser source.

\section{References}

[1] Davis, K.M., et al., Writing waveguides in glass with a femtosecond laser. Optics Letters, 1996. 21(21): p. 1729-1731.

[2] Kondo, Y., et al., Fabrication of long-period fiber gratings by focused irradiation of infrared femtosecond laser pulses. Optics Letters, 1999. 24(10): p. 646-648.

[3] Dragomir, A., et al., Inscription of long-period fibre gratings by femtosecond UV irradiation. SPIE Proceedings, 2003. 4876: p. 313-320.

[4] Mihailov, S.J., et al., Fiber Bragg gratings made with a phase mask and 800-nm femtosecond radiation Optics Letters, 2003. 28(12): p. 995-997.

[5] Mihailov, S.J., et al., Bragg Gratings Written in All$\mathrm{SiO}_{2}$ and Ge-Doped Core Fibres With 800-nm Femtosecond Radiation and a Phase Mask. Journal of Lightwave Technology, 2004. 22(1): p. 94-100.

[6] Bricchi, E. and P.G. Kazansky. Extraordinary stability of femtosecond direct written structures. in Photonics Summer School. 2004. Bath, United Kingdom.

[7] Grobnic, D., et al., Saphire Fibre Bragg Grating Sensor Made Using Femtosecond Laser Radiation for Ultrahigh Temperature Applications. IEEE Photonics Technology Letters, 2004. 16(11): p. 2505-2507.

[8] Schaffer, C.B., A Brodeur and E Mazur, Laserinduce breakdown and damage in bulk transparent materials induced by tightly focused femtosecond laser pulses, Measurement Science and Technology, 2001. 12: p. 1784-1794. 